\title{
Determination of Expected TIGERISS Observations
}

\section{Brian F. Rauch, ${ }^{\dagger}, a, *$ Nathan E. Walsh ${ }^{a}$ and Wolfgang V. Zober ${ }^{a}$ for the TIGERISS Collaboration \\ (a complete list of authors can be found at the end of the proceedings)}

${ }^{a}$ Department of Physics and McDonnell Center for the Space Sciences, Washington University, St. Louis, MO 63130 USA

E-mail: brauch@physics.wustl.edu

We present the method used to estimate the cosmic-ray observations expected for that the TransIron Galactic Element Recorder for the International Space Station (TIGERISS), which is designed to measure the abundances of the rare Ultra-Heavy Galactic Cosmic Rays (UHCR) ${ }_{30} \mathrm{Zn}$ and heavier. TIGERISS uses planes of crossed silicon strip detectors at the top and bottom for charge and trajectory determination and acrylic and aerogel Cherenkov detectors for velocity and charge determination. Instruments are modeled in configurations for the Japanese Experiment Module (JEM) "Kibo" Exposed Facility ( 1.66 $\left.\mathrm{m}^{2} \mathrm{sr}\right)$, as an European Space Agency Columbus Laboratory external payload $\left(\sim 1.16 \mathrm{~m}^{2} \mathrm{sr}\right)$, and as an ExPRESS Logistics Carrier (ELC) experiment $(\sim 1.10$ $\left.\mathrm{m}^{2} \mathrm{sr}\right)$. Differential geometry factors determined for detector orientations within the geomagnetic field over the ISS $51.6^{\circ}$ inclination orbit are used to determine geomagnetic screening. Energy spectra are integrated using the higher of the energies needed to trigger the instrument or penetrate the geomagnetic field for time-weighted bins of geomagnetic latitude, instrument orientation, and incidence angle. Finally, abundances are reduced by the fraction of events calculated to fragment in the instrument.

$37^{\text {th }}$ International Cosmic Ray Conference (ICRC 2021)

July 12 th - 23rd, 2021

Online - Berlin, Germany

\footnotetext{
*Presenter

${ }^{\dagger}$ Research supported by the McDonnell Center for the Space Sciences and the Peggy and Steve Fossett Foundation
} 


\section{Introduction}

Measurement of ultra-heavy Galactic cosmic rays (UHGCR), ${ }_{30} \mathrm{Zn}$ and higher charge elements, with individual element resolution is challenging. Figure 1 shows the relative abundances of elements from ${ }_{1} \mathrm{H}$ to ${ }_{40} \mathrm{Zr}$ for Galactic cosmic rays (GCR) with energies of $2 \mathrm{GeV} /$ nucleon compared with the Solar System (SS) abundances normalized to ${ }_{14} \mathrm{Si}$. These two samples of interstellar matter (ISM) are broadly consistent, with the much younger few million year old GCR filling in many of the valleys seen in the older $\sim 4.6$ billion year old SS, largely from GCR spallation between the source and detection. In the GCR we see that ${ }_{26} \mathrm{Fe}$ is $\sim 5 \times 10^{3}$ times less abundant than ${ }_{1} \mathrm{H}$, and that the UHGCR are $\sim 10^{5}$ times less abundant than ${ }_{26} \mathrm{Fe}$. The abundances continue to fall above ${ }_{40} \mathrm{Zr}$.

The Trans-Iron Galactic Element Recorder for the International Space Station (TIGERISS) is designed to measure the GCR from ${ }_{5} \mathrm{~B}$ to ${ }_{82} \mathrm{~Pb}$ with single-element resoluion to probe the GCR source (GCRS) and the mechanism that injects material into the GCR accelerator. TIGERISS improves upon the preceding TIGER and SuperTIGER instruments by replacing the scintillating fiber hodoscopes and scintillators with silicon strip detectors (SSD), which provide improved charge resolution and allow for both reductions in the material in beam and instrument profile. Event statistics are estimated for TIGERISS based on the method originally derived for and validated with CALET [1] using energy spectra models for the GCR, accounting for geomagnetic screening on the International Space Station (ISS), detector thresholds, and interaction losses in the instrument.

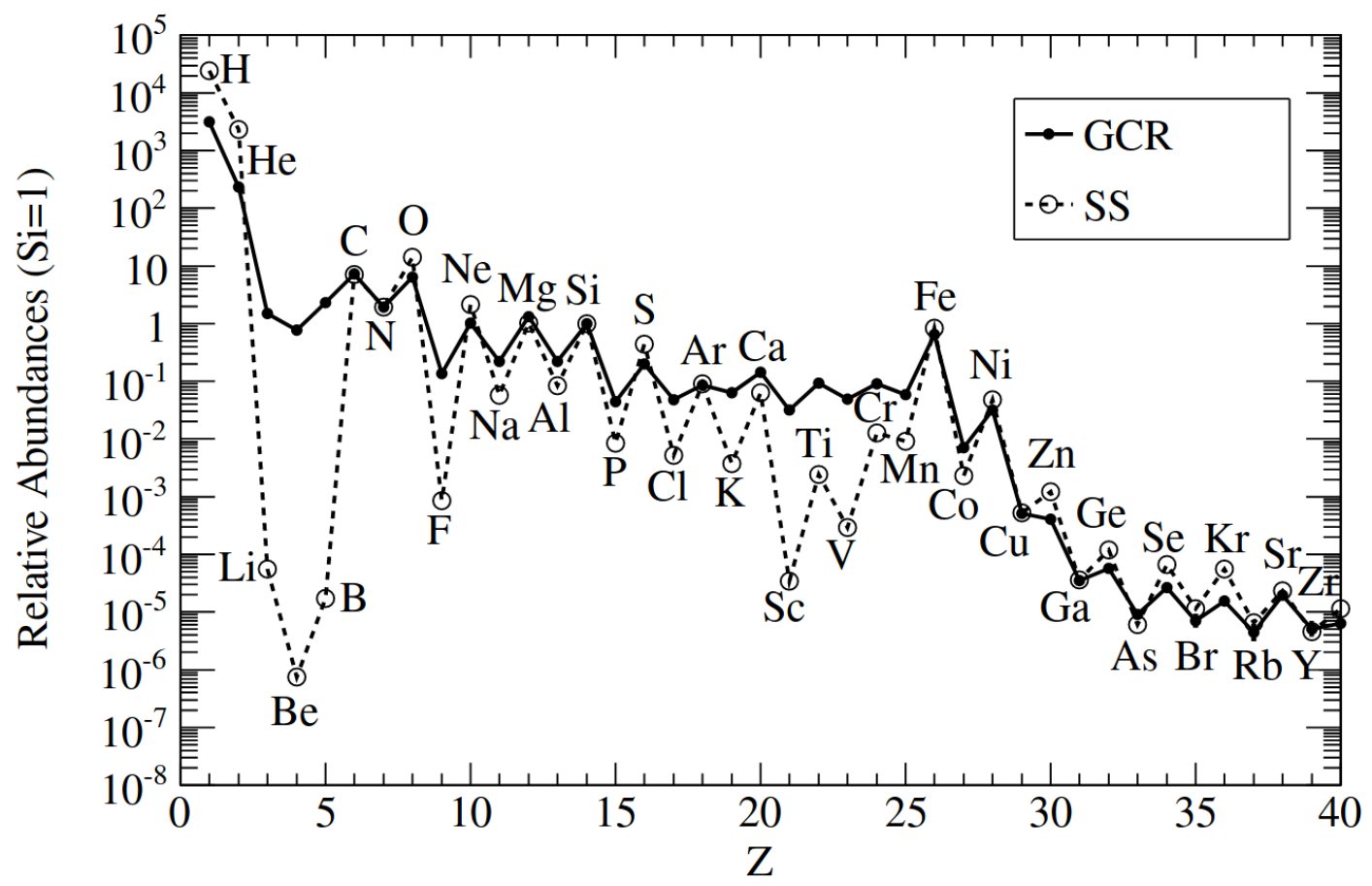

Figure 1: Solar System (SS) [2] and Galactic cosmic-ray (GCR) relative abundances at $2 \mathrm{GeV} /$ nuc. GCR data is sourced for $1 \leq \mathrm{Z} \leq 2$ from [3], $\mathrm{Z}=3$ from [4], $4 \leq \mathrm{Z} \leq 28$ from [5], $\mathrm{Z}=29$ from [6], and $28 \leq \mathrm{Z} \leq 40$ from [7] and normalized to ${ }_{14} \mathrm{Si}$. 


\section{TIGERISS Instrument Models}

TIGERISS has not yet been assigned accommodation on the ISS, so we have developed models for attachment to the Japanese Experiment Module "Kibo" Exposed Facility (JEM-EF), European Space Agency Columbus Laboratory external payload and ExPRESS Logistics Carrier (ELC). A technical model of the JEM-EF configuration shown in Fig. 2a is the baseline for the model calculations presented in this paper. Figure $2 b$ shows this instrument model mounted in a JEM-EF pallet, where detector dimensions are compatible with the ISS-CREAM JEM-EF mounting location. There is ample space for thermal, power and electronics systems below the detector stack.
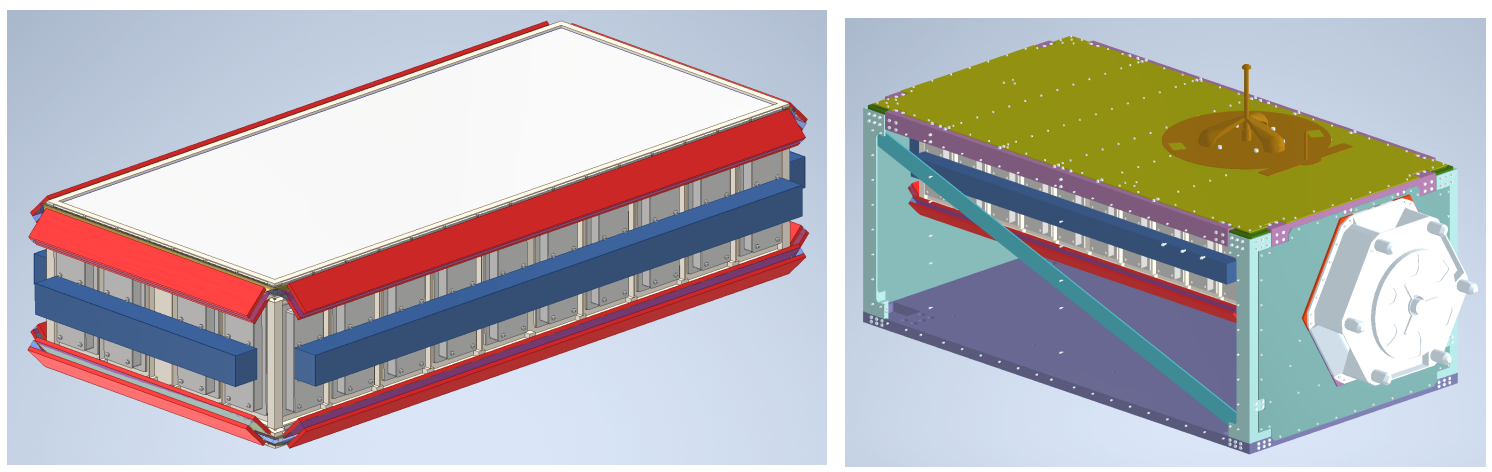

Figure 2(a): Technical model of TIGERISS detector stack with top Figure 2(b): TIGERISS instrument model shown and bottom crossed SSD layers between aerogel (C0) and acrylic mounted in the JEM-EF pallet.

(C1) Cherenkov detectors.

Detailed technical models have not been developed for any of the instrument configurations, so SuperTIGER has been used to scale TIGERISS detector dimensions for the different ISS accommodation models and estimate the instrument materials while accounting for the SSD replacement of scintillator based detectors. SSD arrays at the top and bottom of the instrument measure particle trajectories and ionization energy deposits $(d E / d x)$. Two Cherenkov detectors measure nuclear charge $(Z)$ and velocity $(\beta)$ : $\mathrm{C} 1$ with an acrylic radiator (optical index of refraction $n=1.49, \beta>$ $0.67, \mathrm{KE} \geq 325 \mathrm{MeV} /$ nucleon $)$ and $\mathrm{C} 0$ with a silica aerogel radiator $(n=1.04, \beta>0.96, \mathrm{KE} \geq 2.25$ $\mathrm{GeV} /$ nucleon).

\begin{tabular}{l|l|l|l|l|l} 
ISS attachment & length & width & height & area & geometry factor \\
\hline JEM-EF & $1.67 \mathrm{~m}$ & $0.67 \mathrm{~m}$ & $0.40 \mathrm{~m}$ & $1.12 \mathrm{~m}^{2}$ & $1.66 \mathrm{~m}^{2} \mathrm{sr}$ \\
ELC & $1.05 \mathrm{~m}$ & $0.75 \mathrm{~m}$ & $0.40 \mathrm{~m}$ & $0.79 \mathrm{~m}^{2}$ & $1.10 \mathrm{~m}^{2} \mathrm{sr}$ \\
Columbus & $0.98 \mathrm{~m}$ & $0.75 \mathrm{~m}$ & $0.35 \mathrm{~m}$ & $0.74 \mathrm{~m}^{2}$ & $1.10 \mathrm{~m}^{2} \mathrm{sr}$ \\
Columbus & $0.98 \mathrm{~m}$ & $0.75 \mathrm{~m}$ & $0.32 \mathrm{~m}$ & $0.74 \mathrm{~m}^{2}$ & $1.15 \mathrm{~m}^{2} \mathrm{sr}$
\end{tabular}

Table 1: TIGERISS instrument dimensions and geometry factors.

Geometry factors have been calculated for simple models for all three ISS accommodation options, with details shown in Table 1. Integrated geometry factors from these models are given in Fig. 3a for the JEM-EF, Fig. 3b for the ELC, and Fig. 3c for the Columbus Laboratory TIGERISS configurations, showing that most of the acceptance is within $\sim 60^{\circ}$. Table 1 gives Columbus 
TIGERISS models with different heights, demonstrating that the shorter $0.32 \mathrm{~m}$ tall model shown in Fig. $3 \mathrm{c}$ has a slightly larger geometry factor than the taller $0.35 \mathrm{~m}$ tall model.

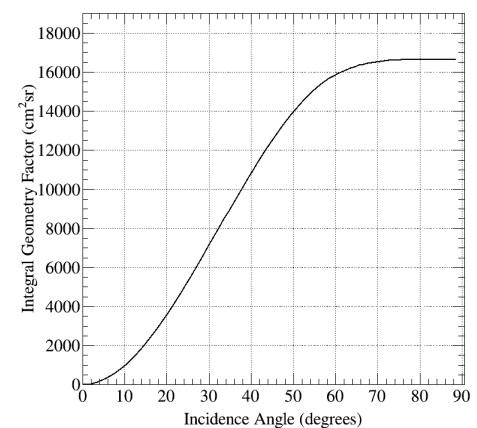

Figure 3(a): JEM-EF configuration: Figure 3(b): ExPRESS Logistics Car- Figure 3(c): ESA Columbus Lab$167.0 \mathrm{~cm}(\mathrm{~L}) 67.0 \mathrm{~cm}(\mathrm{~W}) 40.0 \mathrm{~cm}(\mathrm{~T})$ rier $($ ELC) configuration: $105.0 \mathrm{~cm}(\mathrm{~L})$ oratory external payload configura$\sim 1.66 \mathrm{~m}^{2} \mathrm{sr}$.

\section{Modelling Geomagnetic Screening}

Geomagnetic screening is based on both the strength of the field and the relative orientation of the charged GCR nuclei to it. GCR normally incident at the geomagnetic poles travel along the field lines without resistance while those incident at the equator are most strongly screened. The vertical screening scales with the geomagnetic latitude, and Fig. 4a shows the corresponding vertical cutoff rigidities sampled by the ISS $51.6^{\circ}$ orbit at $\sim 400 \mathrm{~km}$, ranging from $\sim 1$ to $\sim 15 \mathrm{GV}$. The screening threshold strength as a function of GCR trajectory, plotted in Fig. $4 \mathrm{~b}$ relative to the East-West inclination angle $(\gamma)$ and geomagnetic latitude $(\lambda)$, is derived from Equation 1, where $r$ is the distance from Earth's center and $C_{S}$ is the Störmer constant derived from the magnetic dipole moment.

$$
P \geq \frac{1}{r^{2}} C_{S}\left(\frac{1-\sqrt{1-\cos \gamma \cos ^{3} \lambda}}{\cos \gamma \cos \lambda}\right)^{2}
$$

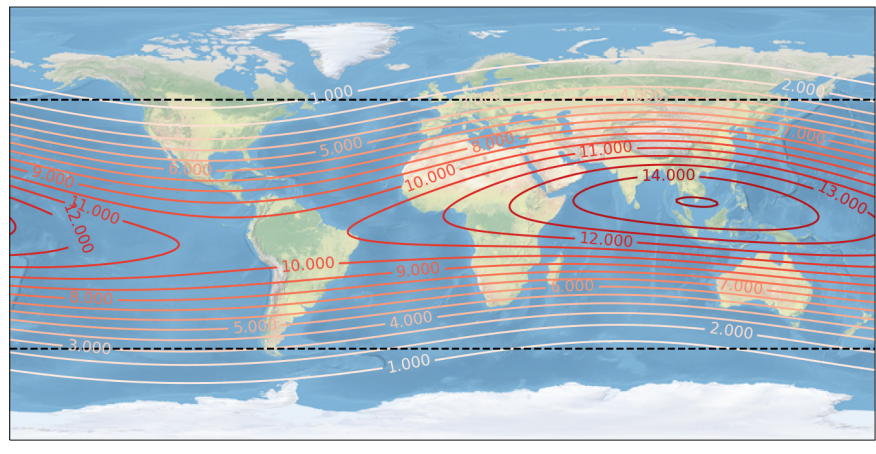

Figure 4(a): Geomagnetic vertical cutoff rigidities sampled by the ISS $51.6^{\circ}$ inclination orbit at $\sim 400 \mathrm{~km}$.

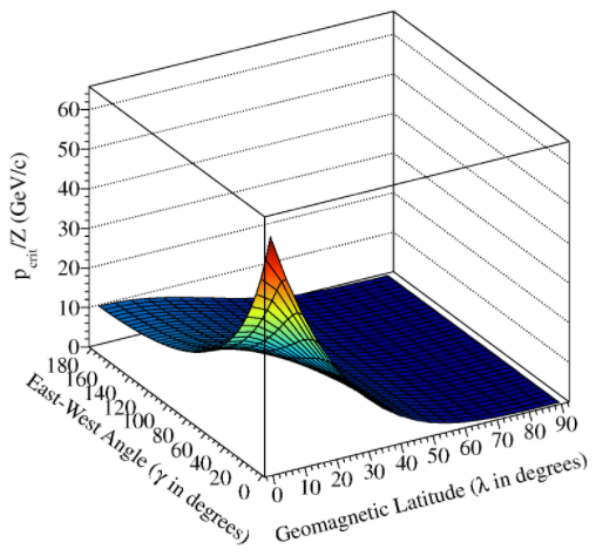

Figure 4(b): Critical momentum to penetrate the geomagnetic field as a function of geomagnetic latitude $(\lambda)$ and East-West angle $(\gamma)$. 


\section{East-West Differential Geometry Factors}

The instrument acceptance for GCR isotropically incident at Earth depends on both instrument geometry and the trajectory dependent geomagnetic screening, and the orientation of the instrument within the geomagnetic field is important. For this analysis we have averaged the expected statistics from cases where the principal axis of the JEM-EF instrument model is aligned with the direction of the geomagnetic field and perpendicular to it, Fig. 5 left and right, respectively. These differential geometry factors are functions of both the incidence $(\theta)$ and East-West $(\gamma)$ angles, mapped in $1^{\circ}$ resolution bins.
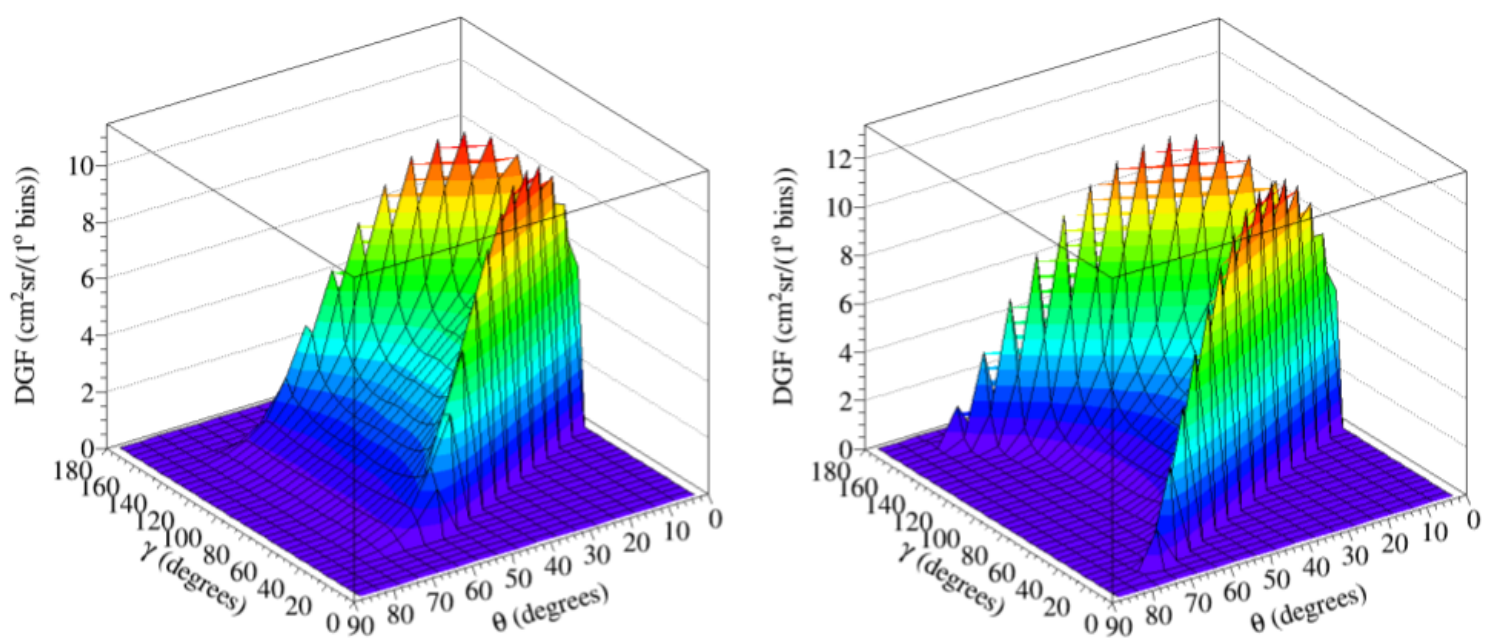

Figure 5: The TIGERISS JEM-EF instrument differential geometry factors as a function of incidence $(\theta)$ and East-West angles $(\gamma)$ for East-West angle aligned with the instrument major (left) and minor (right) axes.

\section{Predicting Abundances}

The orbital residence times at the different vertical cutoff rigidities shown in Fig. 4a are calculated based on the ISS time at the corresponding geographic latitudes and longitudes to find the weighted vertical cutoff rigidities shown in Fig. 6a. Minimum energy thresholds as a function of East-West angle are derived from the trajectory dependent critical momentum (Eq. 1). The higher of the rigidity minimum energy or detector $(\sim 350 \mathrm{Mev} / \mathrm{nuc})$ threshold is used to estimate statistics.

\subsection{Estimating GCR Spectra}

The spectra of elements above ${ }_{28} \mathrm{Ni}$ have not been measured in the GCR, so these have to be estimated based on measured or assumed relative abundances. The UHGCR are mostly primary in composition, so their spectra are derived by scaling the ${ }_{26} \mathrm{Fe}$ spectrum with relative abundances: HEAO-3-C2 for $Z \leq 26$ [5], TIGER for $26 \leq Z \leq 40$ [6], and HEAO-3-HNE for $Z>40$ [8]. TIGERISS is expected to see intermediate to maximum Solar modulation during its mission, so integral spectra derived from differential spectra in [9] for Solar minimum and maximum are used, as well as averaged spectra. 


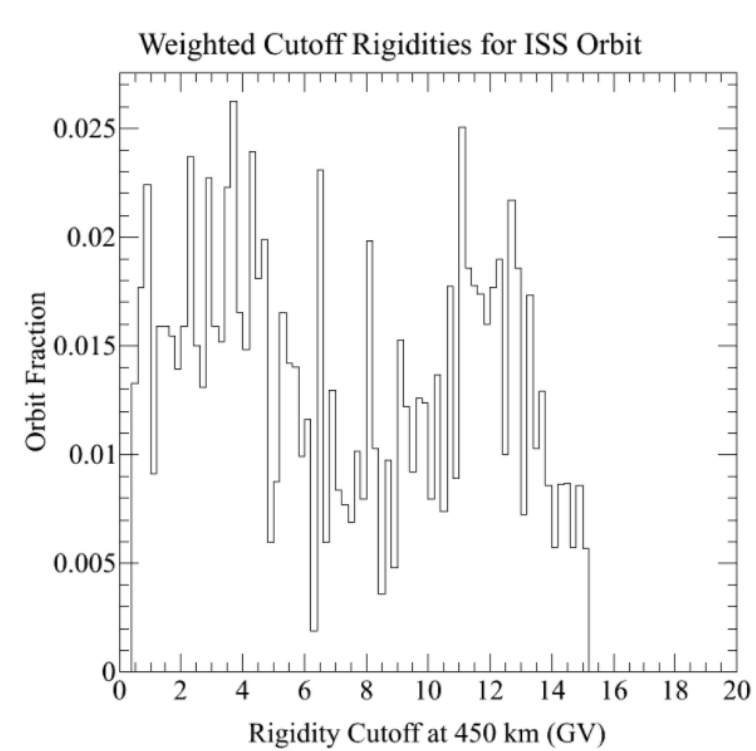

Figure 6(a): The fraction of the ISS orbit spent at each vertical cutoff rigidity.

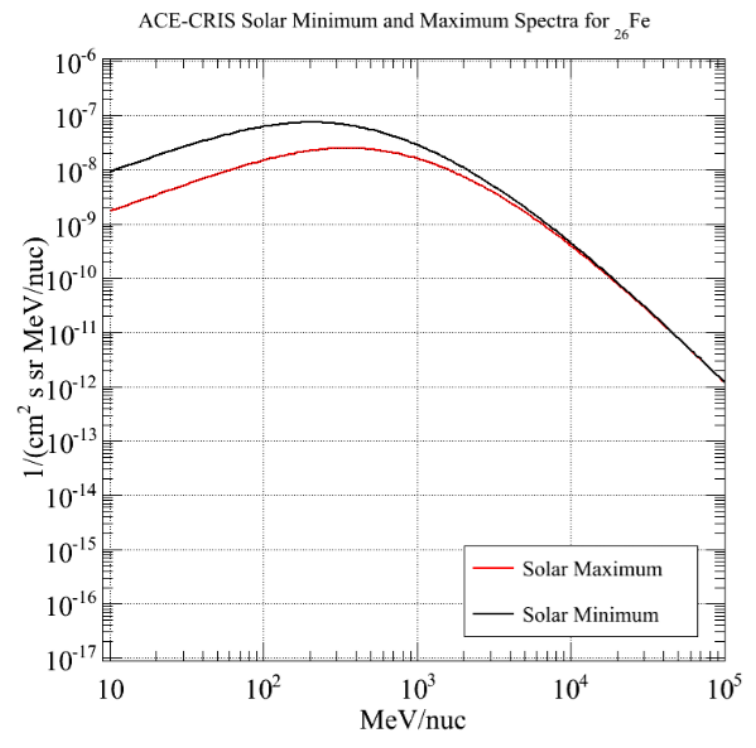

Figure 6(b): Solar maximum and minimum ${ }_{26} \mathrm{Fe}$ differential energy spectra that are integrated and scaled using relative abundances of heavier elements.

\subsection{Estimating Statistics}

The abundances that TIGERISS will see for each element are estimated utilizing their integral spectra to find the events expected at each $1^{\circ}$ geomagnetic latitude $(\lambda)$ step as a function of each $1^{\circ}$ East-West angle $(\gamma)$ step. At each $\gamma$ the integral spectra of each element are evaluated with the greater of the the $325 \mathrm{MeV} /$ nucleon acrylic Cherenkov threshold or the kinetic energy corresponding to the critical momentum: $E_{c r i t}=\sqrt{p_{c r i t}^{2} / A^{2}-m_{a m u}}-m_{\text {amu }}$, where $A$ is the atomic mass, $p_{c r i t}$

\begin{tabular}{l|l|l|l|l}
$\begin{array}{l}\text { detector } \\
\text { material }\end{array}$ & $\begin{array}{l}\text { chemical } \\
\text { formula }\end{array}$ & $\begin{array}{l}\text { thickness } \\
(\mathrm{cm})\end{array}$ & $\begin{array}{l}\text { density } \\
\left(\mathrm{g} / \mathrm{cm}^{3}\right)\end{array}$ & $\begin{array}{l}\text { areal density } \\
\left(\mathrm{g} / \mathrm{cm}^{2}\right)\end{array}$ \\
\hline aluminum & $\mathrm{Al}$ & 0.168 & 2.700 & 0.454 \\
silicon & $\mathrm{Si}$ & 0.400 & 2.329 & 0.932 \\
PET & $\mathrm{C}_{10} \mathrm{H}_{8} \mathrm{O}_{4}$ & 0.090 & 1.135 & 0.102 \\
PMI & $\mathrm{C}_{8} \mathrm{H}_{11} \mathrm{O}_{2} \mathrm{~N}$ & 8.732 & 0.032 & 0.279 \\
PMMA & $\mathrm{C}_{5} \mathrm{H}_{8} \mathrm{O}_{2}$ & 1.270 & 1.180 & 1.499 \\
Kapton & $\mathrm{C}_{22} \mathrm{H}_{10} \mathrm{~N}_{2} \mathrm{O}_{5}$ & 0.0127 & 1.420 & 0.0180 \\
silica & $\mathrm{SiO}_{2}$ & 2.000 & 0.205 & 0.410 \\
PE & $\mathrm{C}_{2} \mathrm{H}_{4}$ & 0.006 & 0.919 & 0.00552 \\
PU & $\mathrm{C}_{25} \mathrm{H}_{42} \mathrm{~N}_{2} \mathrm{O}_{6}$ & 0.635 & 0.080 & 0.0508 \\
PTFE & $\mathrm{C}_{2} \mathrm{~F}_{4}$ & 0.100 & 0.600 & 0.060
\end{tabular}

Table 2: TIGERISS instrument model materials: polystyrene (PS), aluminum (Al), silicon ( $\mathrm{Si}$ ), polyethylene terephthalate (PET) - Mylar, polymethacrylimide (PMI), polymethyl methacrylate (PMMA) - acrylic, Kapton (polyimide film), polyethylene (PE), polyurethane (PU), polytetrafluoroethylene (PTFE) 
is the critical momentum, and $m_{a m u}$ is the atomic mass unit mass. These fluxes are multiplied by the differential geometry factors shown in Fig. 5 that are a function of $\gamma$ and zenith angle, $\theta$, and are then averaged. The fluxes for each element are then reduced by the fraction of events that would interact in the active area of the instrument. This is done using total charge changing cross sections given by $\sigma_{\text {tot }}(P, T)=\pi\left[R_{P}+R_{T}-(3.20 \pm 0.05)\right]^{2}$, where $P$ and $T$ refer to the projectile and target nuclei, and $R_{P}$ and $R_{T}$ are their respective nuclear radii [10] for the material areal densities listed in Table 2. Finally, the numbers of each element expected are found by multiplying the non-interacting fluxes by the total observation time of one year and the orbit fraction shown in Fig. 6a.

\section{Results and Discussion}

The elemental abundances predicted for the TIGERISS JEM-EF instrument after one year of operation on the ISS at average and maximum Solar activity are compared to those measured by SuperTIGER during its 55 day long-duration-balloon flight $[11,12]$ in Fig. 7. The TIGERISS statistics are not strongly dependent on the level of Solar modulation because the geomagnetic screening in the ISS orbit limits the lower energy nuclei most strongly affected by modulation. The one year TIGERISS results are comparable to those of SuperTIGER and would be free of the systematic effects from the corrections needed to account for atmospheric nuclear interactions and energy losses. The UHGCR statistics from one year would be about half of what HEAO-3-HNE observed, but with single-element resolution through ${ }_{82} \mathrm{~Pb}$ would have significant exploratory value.

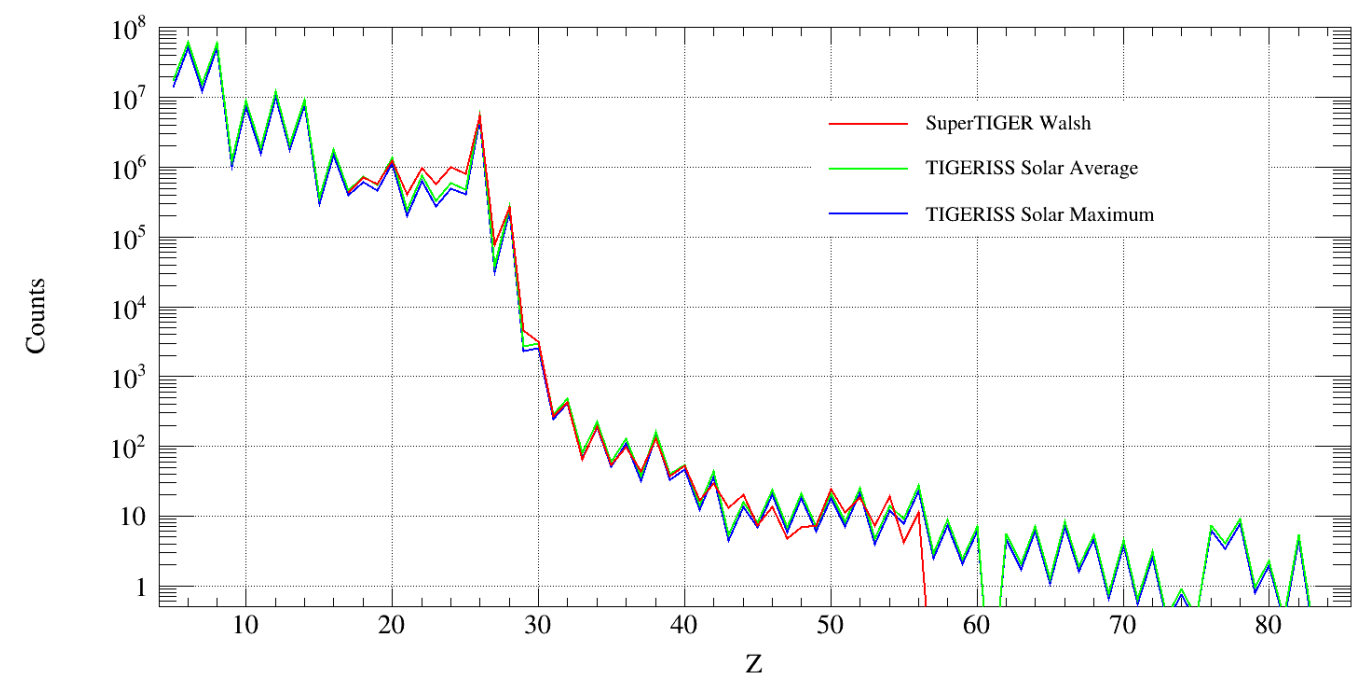

Figure 7: Predicted abundances measured by TIGERISS after 1 year of operation compared to those measured by SuperTIGER over its 55 day long-duration-balloon flight [11, 12]

There are a number of improvements we plan to make to these predictions in the near future. First, we will develop improved instrument models for each of the three ISS attachment points with the largest detector geometries possible accounting for structural requirements. These models will also include the specific materials and thicknesses that would be used for an instrument on the ISS. The interaction corrections will be expanded to include corrections to element fluxes based 
on the inactive material at the top of the instrument, accounting for both gains and losses to each element. An energy loss calculation will be implemented to allow us to more accurately model the incidence-angle dependent minimum detector threshold for nuclei to register in the bottom SSD layer rather than the more conservative acrylic Cherenkov threshold currently used. Finally, we will implement differential geometry factors for each orientation of the detector with respect to the East-West angle weighted appropriately for orbit fractions.

\section{References}

[1] B. F. Rauch for the CALET Collaboration, "Predicted CALET measurements of ultra-heavy cosmic ray relative abundances," Advances in Space Research, vol. 53, pp. 1444-1450, May 2014.

[2] K. Lodders, "Solar System Abundances and Condensation Temperatures of the Elements," The Astrophysical Journal, vol. 519, pp. 1220-1247, 2003.

[3] T. Sanuki et al., "Precise Measurement of Cosmic-Ray Proton and Helium Spectra with the BESS Spectrometer," The Astrophysical Journal, vol. 545, no. 2, pp. 1135-1142, 2000.

[4] M. Aguilar et al., "Isotopic Composition of Light Nuclei in Cosmic Rays: Results from AMS-01,” The Astrophysical Journal, vol. 736, p. 105, Aug. 2011.

[5] J. J. Engelmann et al., "Charge Composition and Energy Spectra of Cosmic-Ray Nuclei for Elements from Be to Ni. Results from HEAO-3-C2," Astronomy \& Astrophysics, vol. 233, pp. 96-111, 1990.

[6] B. F. Rauch et al., "Cosmic Ray Origin in OB Associations and Preferential Acceleration of Refractory Elements: Evidence from Abundances of Elements ${ }_{26} \mathrm{Fe}$ through ${ }_{34} \mathrm{Se}$," The Astrophysical Journal, vol. 697, no. 2, pp. 2083-2088, 2009.

[7] R. P. Murphy et al., "Galactic Cosmic Rays Origins and OB Associations: Evidence from SuperTIGER Observations of Elements ${ }_{26} \mathrm{Fe}$ through ${ }_{40} \mathrm{Zr}$," The Astrophysical Journal, vol. 831, no. 2, p. 148, 2016.

[8] W. R. Binns et al., "Abundances of Ultraheavy Elements in the Cosmic Radiation: Results from HEAO 3," The Astrophysical Journal, vol. 346, pp. 997-1009, 1989.

[9] J. S. George et al., "Elemental Composition and Energy Spectra of Galactic Cosmic Rays During Solar Cycle 23," The Astrophysical Journal, vol. 698, no. 2, 2009.

[10] B. S. Nilsen et al., "Fragmentation Cross Sections of Relativistic ${ }_{36}^{84} \mathrm{Kr}$ and ${ }_{47}^{109} \mathrm{Ag}$ Nuclei in Targets From Hydrogen to Lead," Physical Review C, vol. 52, no. 6, pp. 3277-3290, 1995.

[11] N. E. Walsh, SuperTIGER Elemental Abundances for the Charge Range $41 \leq Z \leq 56$. PhD thesis, Washington University in St. Louis, 2020.

[12] N. E. Walsh et al., "SuperTIGER Abundances of Galactic Cosmic Rays for the Atomic Number (Z) Interval 30 to 56," in Proceedings of 37th International Cosmic Ray Conference - PoS(ICRC2021), vol. 395, p. 118, 2021. 


\section{Full Authors List: TIGERISS Collaboration}

M. Alfred ${ }^{1}$, M. Amoo ${ }^{2}$, T. Anderson ${ }^{3}$, R. G. Bose ${ }^{4}$, T. J. Brandt ${ }^{5}$, J. H. Buckley ${ }^{4}$, N. Cannady $6,7,8$, R. Caputo ${ }^{5,7}$,

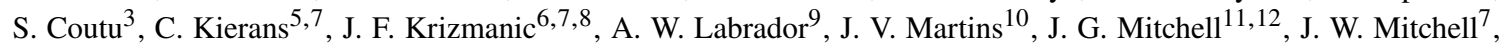
S. A. I. Mognet ${ }^{3}$, A. A. Moiseev ${ }^{7,8,13}$, G. A. de Nolfo ${ }^{12}$, S. Nutter ${ }^{14}$, B. F. Rauch ${ }^{4}$, K. Sakai ${ }^{6,7,8}$, M. Sasaki ${ }^{7,8,13}$, N. E. Walsh ${ }^{4}$, L. P. Williams ${ }^{6,7,8}$, and W. V. Zober ${ }^{4}$

${ }^{1}$ Department of Electrical Engineering and Computer Science, Howard University, Washington, DC 20059, USA

${ }^{2}$ Department of Physics and Astronomy, Howard University, Washington, DC 20059, USA

${ }^{3}$ Department of Physics, Penn State University, University Park, PA 16802, USA

${ }^{4}$ Department of Physics and McDonnell Center for the Space Sciences, Washington University, St. Louis, MO 631304899, USA

${ }^{5}$ NASA Goddard Space Flight Center, Greenbelt, MD 20771, USA

${ }^{6}$ Center for Space Sciences and Technology, University of Maryland Baltimore County, Baltimore, MD 21250, USA

${ }^{7}$ Astroparticle Physics Laboratory, NASA/GSFC, Greenbelt, Maryland 20771, USA

${ }^{8}$ Center for Research and Exploration in Space Sciences and Technology, NASA/GSFC, Greenbelt, MD 20771, USA

${ }^{9}$ California Institute of Technology, Pasadena, CA 91125, USA

${ }^{10}$ Department of Physics, University of Maryland Baltimore County, Baltimore, MD 21250, USA

${ }^{11}$ Department of Physics, The George Washington University, Washington, DC 20052, USA

${ }^{12}$ Heliospheric Physics Laboratory, NASA/GSFC, Greenbelt, MD 20771, USA

${ }^{13}$ Department of Astronomy, University of Maryland, College Park, MD 20742, USA

${ }^{14}$ Dept. of Physics and Geology, Northern Kentucky University, Newport, KY 41099, USA 